\title{
Germanica
}

\section{Ingeborg Bachmann. Engendrer avec les mots Genre ou Geschlecht?}

Ingeborg Bachmann. Mit den Worten erzeugen. Gender oder Geschlecht?

Engendering with the words Gender and Geschlecht.

\section{Françoise Rétif}

\section{OpenEdition}

Journals

Édition électronique

URL : http://journals.openedition.org/germanica/1052

DOI : 10.4000/germanica.1052

ISSN : 2107-0784

Éditeur

Université de Lille

Édition imprimée

Date de publication : 30 juin 2010

Pagination : 73-86

ISBN : 9782913857254

ISSN : 0984-2632

\section{Référence électronique}

Françoise Rétif, «Ingeborg Bachmann. Engendrer avec les mots Genre ou Geschlecht ? », Germanica

[En ligne], 46 | 2010, mis en ligne le 01 juin 2012, consulté le 06 octobre 2020. URL : http:// journals.openedition.org/germanica/1052 ; DOI : https://doi.org/10.4000/germanica.1052

Ce document a été généré automatiquement le 6 octobre 2020.

(c) Tous droits réservés 


\title{
Ingeborg Bachmann. Engendrer avec les mots Genre ou Geschlecht?
}

\author{
Ingeborg Bachmann. Mit den Worten erzeugen. Gender oder Geschlecht? \\ Engendering with the words Gender and Geschlecht.
}

\section{Françoise Rétif}

1 «Ich pflanze mich fort mit den Worten und ich pflanze auch Ivan fort, ich erzeuge ein neues Geschlecht, aus meiner und Ivans' Vereinigung kommt das Gottgewollte in die Welt $»^{1}$. Cette phrase, extraite du premier chapitre du roman Malina, pourrait être placée en exergue de l'œuvre d'Ingeborg Bachmann², car elle est emblématique de la position tout à fait originale de l'écrivaine autrichienne dans un débat qui traverse la deuxième moitié $\mathrm{du} \mathrm{xx}^{\mathrm{e}}$ siècle et dans lequel elle inscrit une conception irréductible à toute autre, exceptionnellement originale et novatrice, encore à notre époque, ne serait-ce que parce que s'y trouvent mêlées trois problématiques centrales de son œuvre : la nécessité de renouveler la pensée des catégories figées de l'humain, en particulier du féminin et du masculin, sans toutefois s'abstraire du Geschlecht; la transgression des catégories des genres littéraires - ce n'est pas un hasard si ce passage en prose débouche dans le roman sur une envolée lyrique- et la réécriture des mythes.

2 Le mot «Geschlecht », dont la palette polysémique n'a pas d'équivalent en français, joue dans la phrase citée ci-dessus un rôle déterminant. Couplé aux verbes «fortplanzen/reproduire » et " erzeugen/engendrer », le mot s'impose immédiatement dans son sens premier de sexe lié à la fonction biologique de l'engendrement d'une " espèce »; mais c'est aussi, en rapport avec Dieu, un terme fortement connoté par le langage biblique et le mythe du premier homme et de la première femme, dans ce sens il signifie également "lignée, descendance» et convoque une autre œuvre de Bachmann où cette thématique de l'inscription de l'individu dans la longue généalogie humaine est centrale: la nouvelle Tout/Alles. Le lien établi entre ce terme et la thématique du langage ( les mots ») évoque par ailleurs les écrits et termes bibliques (" das Buch von des Menschen Geschlecht»); ce lien est toutefois au plus haut point paradoxal, puisqu'il s'agit ici non pas seulement d'écrire le livre d'un nouveau " genre » humain, mais de l'engendrer avec des mots. Il s'agit d'allier en quelque sorte la création 
par le Verbe (ainsi que Dieu créa Adam) à la (pro)création par le sexe (ainsi qu'Adam et Ève engendrèrent leurs descendants). On est ainsi en présence d'une sorte d'oxymore apparemment innocent mais en fait au plus haut point iconoclaste, condensant un débat théorique et polémique explosif, celui de l'opposition de la création et de la procréation, du naturel et du culturel, du sexe et du genre (gender). Dans cette perspective, la traduction de "Geschlecht", qui, on l'a dit, n'a pas d'équivalent français, apparaît comme particulièrement redoutable ${ }^{3}$. Le champ sémantique de " sexe » est beaucoup plus limité que celui de «Geschlecht»; il en est de même pour " espèce "; le mot français le plus polysémique, et donc le plus adapté pour rendre la charge théorique du passage, est genre ${ }^{4}$. La connotation " genre littéraire », absente du mot allemand « Geschlecht ", loin de faire violence au texte, met au contraire en relief le fait que le passage lui-même, et le roman-opéra Malina dans son ensemble, est à la fois une réflexion sur la transgression des genres littéraires et sa mise en pratique. Le plus gênant dans le choix du mot "genre" dans ce cas est que ce terme désigne désormais aussi, à l'instar du gender venu tout droit de l'Amérique des années 1970-805, le « sexe social ", c'est-à-dire le sexe culturel par opposition au sexe naturel ; il est donc connoté à l'opposé de "Geschlecht", c'est-à-dire du mot utilisé par Bachmann, puisqu'il correspond à la tendance actuelle qui tend à privilégier la culture sur la nature jusqu'à nier la part de la nature dans la sexualité. Notons que les féministes allemandes, dans leur grande majorité, se sont résolues assez récemment à parler de "Gender Studien " plutôt que de « Geschlechterforschung ». L'on voit qu'en l'espace de quelques mots (et du problème de leur traduction) se trouvent condensées une problématique et une polémique qui est au plus haut point d'actualité quand Bachmann publie Malina, en 1973, sans pour cela qu'elles soient désuètes aujourd'hui, quarante ans après.

3 Engendrer un nouveau « Geschlecht »/genre (y compris genre humain) avec des mots, c'est pour Bachmann fustiger la tradition, la reproduction du même, mais ne pas rompre avec elle : il s'agit de la réécrire. Toutefois l'avènement du nouveau ne renonce pas à la reproduction biologique désirée dans l'amour entre un homme et une femme, en l'occurrence Moi et Ivan, puisqu'il y a bien union («Vereinigung»). Dénoncer les défauts de la réalité existante et en esquisser une autre possible ne signifie pas couper avec la généalogie. Mais il faut en inventer une nouvelle forme, imprévisible, ouverte, située à la fois dans et au-delà de l'opposition nature/culture, puisqu'elle est issue de l'alliance des mots et de l'amour, des mots de l'amour adressés à Ivan dans ce livre qui ne sera jamais achevé mais qui est esquissé dans le rêve de « ce que Dieu a voulu » mais n'a jamais réalisé - dans ce que ne peut réaliser que cette «nouvelle Ève » ou « Ève future » écrivaine de son amour : l'alliance de la sexualité et du verbe, de l'eau et du feu, du feu et de la pierre, de l'instant et de l'éternité :

Oiseaux de feu

Azurites

Flammes jaillissantes

Gouttes de jade ${ }^{6}$

Bachmann n'a jamais renoncé à la charge sexuelle, biblique et symbolique du mot «Geschlecht ». En des temps où règnent la « déconstruction » et la queer theory, il est nécessaire de le rappeler. Mais cela ne l'a pas empêchée de rêver, comme dans la nouvelle Un pas vers Gomorrhe d'un monde qui ne serait «ni le règne des hommes, ni le règne des femmes ${ }^{7}$, d'une révolution qui ne peut se faire sans prendre conscience que «tout est une question de langage $»^{8}$, qu'il ne peut y avoir « de monde nouveau sans 
nouveau langage $»^{9}$ et que la transgression des catégories étroites de sexe et de sexe social, ne peut être réalisée sans mettre en question les formes symboliques dans lesquelles se constitue, s'exprime et se raconte l'identité, en particulier les genres littéraires et les mythes, ces derniers relevant de diverses traditions et de leur panachage, gréco-romaines, européennes, germaniques, bibliques, etc. ${ }^{10}$

Son œuvre dans son ensemble oscille sans cesse entre Geschlecht et gender, entre construction et déconstruction de la différence des sexes, entre dénonciation et utopie, sans doute en partie au moins pour déjouer les tentatives d'enfermer sa pensée dans une catégorie. Féministe avant l'heure par sa pensée, elle ne s'engagea jamais publiquement auprès de ce mouvement, ce sont plutôt, au contraire, ses critiques qu'elle fit entendre, en tout cas elle ne prit jamais parti pour telle ou telle chapelle. Comme elle le souligna elle-même dans une interview, elle ne s'est jamais laissée " corrompre par l'actualité " ${ }^{11}$; c'est ce qui lui a permis de voir beaucoup plus loin et d'anticiper l'avenir, tout en prévenant les dangers qu'il réservait. C'est ainsi que, tout en préfigurant une certaine déconstruction de la différence des sexes, elle n'a jamais cessé de stigmatiser l'oppression des femmes par les hommes et les abus du système patriarcal. Et tout en réclamant l'égalité des femmes, ou en fustigeant des institutions comme le mariage, elle s'est élevée contre l'identification des femmes aux hommes ou aux modèles masculins, ainsi que contre le renoncement à certaines valeurs traditionnellement «féminines » comme l'amour (qui pour elle cependant n'avait rien à voir avec la conception bourgeoise dominante) sans pour autant défendre des positions essentialistes. Sa position est unique, inclassable : elle varie sans cesse les points de vue, les passant en revue sans jamais se fixer définitivement sur tel ou tel, insouciante des prises de position dominantes ou à la mode, critiquant, bifurquant, inventant, inventant ne serait-ce que par la structure fluide de sa pensée. $\mathrm{Ni}$ construction ni déconstruction, sa déconstruction consiste peut-être en cette oscillation de la pensée qui ne laisse pas fixer, sinon dans le refus de la violence et de l'inégalité entre les sexes.

6 Ingeborg Bachmann en effet n'attendit pas la fin des années soixante pour clouer au pilori les inégalités et les différences abyssales entre les sexes véhiculées par la société, dans la conception de l'amour en particulier. Dans ses pièces radiophoniques, en particulier dans Le Bon Dieu de Manhattan (1958), elle montra l'impuissance à aimer de l'homme et, à l'inverse, la femme victime de son amour passionné. Cette réécriture voilée du mythe de Tristan et Iseult inverse les données traditionnelles ${ }^{12}:$ Jan (alias Tristan) ne perd jamais le sens des réalités et rejoint à temps son pays et sa famille en abandonnant Jennifer (alias Iseult) aux foudres du Bon dieu de Manhattan (alias le Roi Marc), sorte de juge suprême, garant de l'ordre établi, qui la sacrifie sur l'autel du principe de réalité. À l'inverse du mythe, Iseult meurt seule, délaissée par Tristan. Comme dans la tradition mythique par contre, l'amour, cet «autre état » qu'est la passion, cette transgression de frontière qu'elle représente, sont mis au ban de la société pour déranger, déstabiliser, voire mettre en péril un certain ordre social, qui s'attache à maintenir les sentiments dans les limites raisonnables du mariage et des conventions. Là où, à l'origine du mythe littéraire, la frontière séparait les amants de la société en place, le fossé se creuse, dans la pièce radiophonique de 1958, entre les deux amants. L'homme trahit l'amour en trahissant la femme. Il s'est inféodé à un certain modèle de vie qui a abaissé l'amour au rang des divertissements au profit d'investissements plus immédiatement utiles, profitables, lucratifs. Si la femme est 
victime et non héroïne, si son rôle peut paraître effacé, il n'en reste pas moins que c'est elle, et elle seule, qui reste fidèle à la valeur subversive de l'amour telle qu'elle fut représentée à l'origine du mythe, dans les romans «d'aventure» médiévaux. Sa passivité doit donc être considérée comme une forme de résistance. L'inscription de la répartition traditionnelle des rôles sexués que l'on a pu reprocher à Bachmann dans certains cas, en particulier dans cette pièce, est moins innocente qu'il n'y paraît : c'est la valeur subversive de la passion qui est affirmée et donc la force des valeurs féminines. Le « Geschlecht » retrouve son pouvoir dérangeant.

7 C'est bien différemment que Charlotte, dans la nouvelle Un Pas vers Gomorrhe du recueil La Trentième année, tente d'échapper à la mise sous tutelle de la sexualité dans l'ordre patriarcal du mariage. En envisageant la possibilité d'une liaison homosexuelle, elle prend conscience de tout ce qui manque à l'affirmation de son identité et qu'elle ne peut continuer de vivre dans le carcan d'une structure et d'une image imposées. Elle songe un moment à inverser les rôles, à faire de son amie, Mara, "sa proie", sa " créature ", à l'instar du comportement de son mari envers elle-même. On a pu montrer que cette nouvelle est une sorte de réécriture d'un roman de Simone de Beauvoir, L'Invitée (1943) ${ }^{13}$, dans lequel l'écrivaine et philosophe française thématisait ou semblait thématiser la conception sartrienne de l'Autre comme «aliénation subtile de toutes mes possibilités $»^{14}$, la théorie du conflit inévitable des consciences. Dans un texte manuscrit en effet, Bachmann s'en prend aux femmes qui «pensaient dans les catégories des hommes, ces catégories dégradées et simplifiées, un monde où "l'autre" devenait "l'Autre", la diablesse, le pauvre diable, la séductrice légendaire, une pauvre fille, une sœur dans le meilleur des cas $»^{15}$. L'écrivaine autrichienne montre explicitement que le comportement de Charlotte est la conséquence de la situation d'aliénation qu'elle a connue dans le mariage et que, à ce titre, il ne relève pas du prétendu conflit originel des consciences évoqué par Beauvoir dans Le Deuxième Sexe Beauvoir se servant de la théorie de Hegel, importée en France par Kojève, pour d'ailleurs aussitôt la détourner, ce qui échappa à Bachmann ${ }^{16}$. L'héroöne bachmannienne, contrairement à celle de Beauvoir, ne se résout pas à inverser les rôles; elle ne fera pas de Mara sa proie, et elle ne fera pas le pas décisif vers l'homosexualité. Toutefois, elle a fait «un pas » vers la libération; elle se met à rêver d'un monde dans lequel n'existeraient, comme nous l'avons déjà souligné, «ni le règne des hommes ni le règne des femmes " et où l'on "ne naîtrait pas prisonnier d'une image ». En d'autres termes, elle se refuse à franchir le seuil qui la mènerait à la reproduction des comportements masculins, tout en prenant ses distances par rapport à certains comportements féminins : la nouvelle contient une critique corrosive d'une certaine "parole de femme » ou d'une certaine hystérie féminine. Entre universalisme et essentialisme, Bachmann tend déjà vers la déconstruction des catégories préétablies des comportements et rôles sociaux de sexe qui trouvera son aboutissement dans le poème de 1964, La Bohême est au bord de la mer. Mais le rêve d'un monde où chacun pourrait être soi-même sans devoir obéir à une norme sexuelle (ou autre) n'a jamais permis que soient oubliées les violences qui régissent les rapports de sexe; en cela, Ingeborg Bachmann a anticipé sur le danger potentiel d'une déconstruction du gender qui ne ferait qu'occulter une nouvelle forme de pouvoir machiste. C'est seulement dans l'utopie que peut être envisagé un monde où l'opposition des sexes n'existerait plus. De façon significative, l'être qui incarne le mieux le "trouble dans le genre » dans une sorte de troisième genre, inconnu, indécis, Ondine, qui est de sexe féminin sans être une femme - puisqu'elle est un esprit élémentaire, une nymphe - est aussi celui qui 
tient le discours critique le plus virulent envers les hommes et le monde civilisé qu'ils incarnent. Cependant, de guerre lasse, elle finit par s'en aller...

8 L'Ondine de Bachmann ${ }^{17}$ doit être située dans une longue tradition germanique qui remonte au moins au romantisme : on le sait, son texte est une réécriture du célèbre conte de Friedrich de la Motte Fouqué18. Cependant l'histoire de cette ondine "naturalisée » allemande s'inscrit elle-même dans l'histoire d'un très vieux mythe européen, celui des sirènes. À l'origine, les sirènes étaient des êtres mi-femmes mioiseaux et vivaient sur un rocher. Ulysse, comme nous le raconte Homère dans L'Odyssée, sortit vainqueur de la confrontation avec les sirènes en neutralisant doublement leur charme, dans l'action et dans le récit qu'il en fit, puisque c'est ainsi qu'il accéda à la qualité d'aède, en s'appropriant leur chant.

Geneviève Fraisse a souligné «le renouvellement de la figure de la sirène après 1800 », qu'elle interprète comme un signe du dérèglement naissant des représentations des $\operatorname{sexes}^{19}$. Avec le personnage mythique de la sirène, le fossé prétendument insurmontable entre corps et esprit, masculin et féminin, nature et culture - et qui n'a cessé de se creuser au long des siècles depuis l'Odyssée, ainsi que l'ont montré Adorno et Horkheimer dans La Dialectique de la raison - ce fossé apparaît pour la première fois dans toute son ambiguïté : la réhabilitation des sirènes dans la littérature allemande de la fin du XVIII et du début du XIX siècle témoigne en effet à la fois du désir et de la peur de le combler.

Ingeborg Bachmann, en orchestrant la voix de la sirène, à la fois inverse et invalide ces dichotomies aussi vieilles que la civilisation (occidentale). L'Ondine de Bachmann incarne en fait une autre loi, sa loi, qui est justement la transgression des frontières et de ce qu'elles représentent. Contrairement aux autres ondines qui la précédèrent, avant de déclarer son départ a priori définitif, elle a transgressé sans relâche, dans les deux sens, de son initiative et de son plein gré, la frontière qui sépare son monde, le monde de l'eau, le monde naturel, du monde des hommes, celui de la civilisation. Elle n'a rien fait d'autre que s'employer à passer de l'eau à la terre et de la terre à l'eau et à aimer; elle va et vient, passe et repasse, sa loi, c'est le passage. Chaque ligne du texte exprime cette logique du passage et de la non-contradiction, non seulement au niveau du contenu, mais aussi dans sa forme: parce que les limites des genres (lyrique, dramatique, épique) sont transgressées; parce que les paroles d'Ondine «nous introduisent dans un monde d'une grande subtilité où se produit l'effacement des charnières d'opposition, et la dissolution des frontières ${ }^{20}$. D'ailleurs l'eau, élément dont vient et provient Ondine, par définition un élément insaisissable, mouvant, imprévisible, sur lequel on ne peut tracer de frontières qu'invisibles et perméables, est pour ce monde comme une métonymie. Ondine aime sa "transparence épaisse $»^{21}$ : l'eau, défie la logique d'opposition binaire des hommes; et Ondine monte sur terre pour la défier aussi. Elle est de l'un et l'autre côté, elle est l'une et l'autre : nymphe, esprit de l'eau, elle appartient au règne naturel. Quand elle est sur terre, femme aimante, elle qui exhorte l'homme à plus de vérité, qui l'incite à penser autrement, qui le mène plus près d'une âme - alors que chez Fouqué, elle venait sur terre pour obtenir, grâce à l'union avec un homme, l'âme qui manque aux êtres naturels - elle incarne au plus haut point la culture, une autre forme de civilisation qui ne tourne pas le dos aux forces magiques de la nature. En Ondine, le corps et l'esprit, la nature et la culture ne font qu'un. En Ondine, l'art, qu'elle incarne, selon les dires mêmes de l'auteur ${ }^{22}$, n'est pas que du côté de la civilisation et de la culture ; il a la mobilité, la fluidité chatoyante, 
toutes les qualités de cet élément naturel qu'est l'eau. Ainsi Ondine, qui n'est pas une femme, mais un esprit élémentaire de sexe féminin, illustre-t-elle l'hybridation fondamentale, par-delà les sexes, de la nature et de la culture dans un art au féminin.

11 Une certaine oscillation entre construction et déconstruction des rôles sexués peut être mise en évidence également dans l'œuvre lyrique. Citons rapidement quelques exemples $^{23}$ : dans le poème Dire l'obscur/Dunkles zu sagen, le Je lyrique, en qui s'incarnent à la fois Orphée et Eurydice (et inversement pour le Tu lyrique), est un exemple de l'indétermination des rôles sexués. Par contre, dans le poème titre Le Temps en sursis / Die gestundete Zeit, on distingue nettement un rôle féminin passif et un rôle masculin actif. Dans le poème Le Pays aîné/Das erstgeborene Land, le Je féminin, mordu comme Eurydice par une vipère, a le pouvoir de se sauver seul en pressant la bouche sur la morsure et c'est la métaphore phallique de la mèche se dressant sous le regard qui clôt le poème. Le poème Prague, janvier 64 / Prag Jänner 64 met en scène la libération et la renaissance d'un Je féminin. Le poème Pas de délicatesses/Keine Delikatessen, qui finit par le vers : «Ma part, cela doit se perdre $»^{24}$, semble convoquer la résignation du Je lyrique identifiable à la poétesse, donc féminin ; cependant étant donné que ce Je n'est pas expressément marqué au féminin (il pourrait tout aussi bien être identifié à un auteur masculin), l'on peut se demander si ce qui doit se perdre (le « es») n'est pas la part subjective du Je, qu'il soit masculin ou féminin, pour arriver à une sorte de voix neutre qui renonce à toute "qualité », telle qu'elle a pu être théorisée par Blanchot ou Barthes $^{25}$. La Bohême est au bord de la mer/Böhmen liegt am Land enfin thématise ce renoncement à toute particularité, entre autres sexuelle, pour la qualité unique et essentielle de "toucher toujours plus à tout $»^{26}$ sur un continuum qui relie non seulement les genres "troubles", mais toute chose existante. Jamais encore avant ce poème, Bachmann n'avait atteint une vision aussi parfaite «du pays de [s]on choix ${ }^{27}$, le pays rêvé où toute lutte serait devenue inutile, où pourrait enfin régner l'absence de « qualités », où serait atteinte une autre origine, engendrée par et dans les mots.

Ce poème est un conte; on y a noté depuis longtemps l'allusion au Conte d'hiver de Shakespeare, dans lequel les didascalies indiquent qu'Antigonus, à la scène 3 de l'acte III, débarque dans « une campagne déserte au bord de la mer ", nommée Bohême, ce qui explique que la mer, dans le poème de Bachmann, soit qualifiée de "contestée " (puisque l'on fit à Shakespeare, à l'époque, le reproche d'avoir fait une erreur). Comme la pièce de Shakespeare, le poème de Bachmann évoque une résurrection, non pas de la mère, mais du Je renaissant après avoir touché le fond. Le merveilleux ici contamine l'espace: le plan vertical dans le resurgissement du Je presque anéanti; le plan horizontal de par la translation de la Bohême vers la mer. On peut y lire aussi comme une vague réminiscence du conte de Dame Holle (Grimm) dans lequel l'initiation de la fille, de la femme consiste à découvrir le pays de Cocagne au fond du puits. J'y entends en outre, dans l'évocation des ponts indemnes, non seulement l'utopie d'un monde sans guerre, mais également une réminiscence d'un autre $\operatorname{Conte}^{28}$ très connu, celui de Goethe, dans lequel la belle Fleur de Lys - relevant à la fois de la sirène et de la Méduse - et le monde entier sont sauvés par le « pont» qui finalement relie les rives; alors la métamorphose est reine : la pierre devient chair, la mort vie, la forme amour, l'amour forme. Pas de métamorphose toutefois, pas de temple, pas de château, pas même de Maison d'Autriche dans le poème de Bachmann: mais de simples «maisons vertes " ${ }^{29}$, habitables, rêve d'un monde démocratique, sans "champignon atomique $»^{30}$, utopie sociale et littéraire d'un univers où régnerait la coexistence pacifique des êtres et des choses, des peuples et des classes, des mots et des êtres. Car les contraires, le Moi et 
l'autre, le féminin et le masculin, ne s'opposent plus; ils ont frontière commune qui relie autant qu'elle distingue. C'est la notion même de frontière qui est redéfinie. Dans le "grenzen" - mot intraduisible en français, qui signifie toucher aux confins, s'approcher de la frontière (c'est donc sans doute la notion de «toucher à » de Jean-Luc Nancy qui en est le plus proche) - la séparation (et toutes les notions corrélatives, l'opposition, la différence, la mort, etc.) n'est pas niée : c'est ce qui lie. La lésion lie. Et la liaison sépare. L'ambiguïté, la conscience de l'ambivalence originelle traverse toute chose et même les mots. Elle s'est érigée en loi, ou plutôt en contre-loi, structurant non seulement le texte littéraire mais aussi la pensée, une autre logique. La frontière est à l'origine et l'origine est au fond. Les radicaux « grenz » et «grund » du texte original font chaînon. Heidegger et Wittgenstein ${ }^{31}$ s'entremêlent. Der Satz vom Grund, (Heidegger, 1957) ${ }^{32}$ est reformulé sur le mode de la variation musicale. «Grund» introduit la répétition du même au niveau du signifiant et simultanément la différence au niveau du signifié à travers des combinaisons changeantes (zugrunde gehen, vom Grund auf, etc.) 33. Le mot "Grund" lui-même se fait frontière liante. Par une série d'anaphores, de répétitions, et par ces variations sur un même signifiant, qui toutes lient et délient en introduisant des glissements successifs au niveau du signifié, le poème avance par translation. Les mots engendrent les mots, une longue lignée de signifiants à la fois semblables et différents; le poème bouge, le poème vit. Au mouvement de la Bohême "graciée » au bord de la mer, répond le mouvement du poème se déroulant grâce aux mots qui font chaîne jusqu'au mot-clé annonçant une réalité jamais atteinte à l'horizon d'un à-venir : la terre espérée, le pays ailleurs, jamais donné, toujours à re-chercher. Ce sont les mots eux-mêmes, qui, rendus à l'espace, entrelacés, accrochés, noués autour de l'écart qui ne cesse de les disjoindre, tressent un méridien qui se déroule sans que rien d'autre ne se construise que ce déroulement incessant vers «le pays de mon choix». L'écriture transporte sans rompre dans un toujours ailleurs; elle véhicule le regard utopique par la transmission du lien.

C'est ainsi que le poème instaure un espace autre, à la fois littéraire, social et philosophique, un espace utopique où tout touche à tout: la mort à la vie, la vie à la mort, le mot à l'être, l'être au mot, l'être à l'autre être dans l'indifférenciation de sexe (même grammatical). Cet espace n'est pas seulement évoqué, il est incarné par et dans le texte lui-même, qui est, dans ses moindres détails, une mise en œuvre de la pensée. Dans cet espace utopique, l'individu singulier, le discours de la singularité humaine n'ont plus cours. L'homme n'est plus défini dans son unité circonscrite au même. En d'autres termes, la notion d'individu, telle qu'elle s'affirme au XvIII ${ }^{\mathrm{e}}$ siècle, est mise en question. "Si c'est moi, c'est tout un chacun qui est autant que moi », ou encore "Si ce n'est pas moi, c'est quelqu'un qui vaut autant que moi ", écrit Bachmann dans $L a$ Bohême. Rousseau écrivait au début des Confessions : «Moi seul : je sens mon cœur et je connais les hommes. Je ne suis fait comme aucun de ceux que j'ai vus; j'ose croire n'être fait comme aucun de ceux qui existent. Si je ne vaux pas mieux, au moins je suis autre $\aleph^{34}$. Dans le monde de Bachmann, l'autre traverse l'identité. Le Moi ne se définit plus par sa singularité, mais par le retrait de sa singularité. Il se définit par sa capacité de passage de l'une à l'autre chose ou être ou mot, en d'autres termes, par sa capacité à "aborder" ou à "toucher" (grenzen) toujours plus à tout. Il est à la fois pluriel et singulier, pas seulement le même et l'autre, mais le même et un pluriel d'autres, le même s'exposant à l'autre, aux autres. "La pluralité de l'étant est au fondement de l'être ", écrit Jean-Luc Nancy dans Être singulier pluriel, en 1996. "Tout l'étant touche à tout l'étant, mais la loi du toucher, c'est la séparation » ${ }^{35}$. Dans La Bohême est au bord de 
la mer, l'être, le Je est déjà « singulier pluriel ». Les identités oscillent, mouvantes. C'est sur les confins qu' « a lieu sans relâche la comparution avec l'autre et devant lui ». Ainsi, « il n'y a pas d'original ni d'origine de l'identité [...]. Ce qui tient lieu d'origine, c'est le partage des singularités. Cela signifie que cette "origine" - l'origine de la communauté ou la communauté originaire - n'est pas autre chose que la limite : l'origine est le tracé des bords sur lesquels, ou le long desquels s'exposent les êtres singuliers $»^{36}$. C'est cette origine-là - une origine réinventée - qui est évoquée par le poème de Bachmann dès 1964. Certes, elle n'est pas théorisée : elle prend forme sous nos yeux, sous nos yeux les mots l'engendrent. Le gender y est déconstruit ; cependant le poème va plus loin : c'est la notion même de Menschengeschlecht qui est relativisée, redéfinie et élargie.

De même, l'œuvre en prose, en particulier les nouvelles de La Trentième année, ne font pas qu'interroger et fragiliser les catégories sexuelles traditionnelles; elles sont déjà au-delà : le gender participe de la remise en question de quelque chose de plus vaste encore que traduit mieux le mot allemand Geschlecht, car ce sont aussi les limites du genre humain, qui sont interrogées dans ces nouvelles, aussi bien par le père de Tout rejetant la généalogie humaine, que par Wildermuth découvrant sa vérité dans l'empathie avec le monde de la nature et des sens, ou encore par le protagoniste de la Trentième année lui-même faisant l'expérience des limites de la pensée et du langage humains. Et cette problématisation simultanée du gender et du Geschlecht va de pair avec la transgression des genres littéraires, en particulier du récit d'enfance et de l'autobiographie. Dans les limites de cette étude, nous ne prendrons qu'un exemple, celui de la nouvelle Tout/Alles ${ }^{37}$.

15 Le père de Tout, loin d'être attaché au monde patriarcal et à ses lois, rêve, plus que tout autre personnage du recueil de nouvelles, de rupture totale avec le passé. Non seulement il se désolidarise de la Loi en l'accusant de tous les maux, mais il veut même, grâce à son fils, "sortir de l'espèce » ("austreten aus dem Geschlecht ») 38, rompre avec toute généalogie, avec le "genre " humain et aussi avec «le sexe " garant de la sempiternelle reproduction des choses et des êtres, de la lignée humaine ininterrompue. Il veut que son fils soit « le premier homme ", que « tout recommence avec lui ", qu'il "sauve le monde ${ }^{39}$. En adéquation avec cette volonté de rupture symbolique, ni lui, ni son fils, ne portent de nom. Excepté cela, le récit d'enfance semble être relativement conforme à la loi du genre : la vie de l'enfant est racontée par le père du début à la fin, de sa naissance à sa mort accidentelle. Toutefois, comme souvent les nouvelles dans l'espace littéraire germanique, ce récit est entouré d'un "cadre " constitué par l'évocation du couple après la mort de l'enfant - un cadre matérialisant en quelque sorte "l'arc de deuil tendu entre un homme et une femme", expression apparaissant elle-même en début et fin de nouvelle ${ }^{40}$. L'homme et la femme, le père et la mère de l'enfant, sont figés dans les rôles sexués traditionnels : d'une part, la mère, aimante, compréhensive, proche de l'enfant, sans exigence, presque sans personnalité ; d'autre part, le père, lointain, méprisant les soins et les gestes d'amour élémentaires, pour ne se préoccuper que du rôle que l'enfant devra tenir dans la société, de sa mission prétendument rédemptrice. Cependant ce père qui veut "sortir du cercle vicieux " de l'existence et de la civilisation, l'incarne en fait plus sévèrement que personne : il n'aime pas son enfant, il le juge, il est son juge, il l'observe " comme un chercheur un cas $»^{41}$, et il est en partie au moins responsable de sa mort. Bachmann se livre donc ici, de façon très subtile, à une sorte de démonstration par l'absurde : le récit traditionnel convient au récit d'une vie achevée, au récit d'une mort - en d'autres termes, le récit convoque la mort, le récit - variation sur Barthes - est une mort. Le père 
a beau rêver de tout changer : il ne fait, comme son fils, que reproduire ce qui existe, au niveau du langage, de la loi et du récit - et même au niveau de la religion, puisqu'il attend de son fils que, comme le Christ, il sauve le monde. Il faut en effet que le fils soit sacrifié, qu'il meure, pour que le père enfin comprenne et accepte de s'inscrire dans la généalogie. Par ses rêves délirants de rupture, le père perpétue la prétendue fatalité du sacrifice qu'il a indirectement provoqué.

La conclusion à tirer est claire : il ne doit pas y avoir de rupture radicale; croire le contraire est une duperie, un leurre qui ne fait que reproduire le même en voulant y échapper. Il faut partir du donné. "C'est dans la confrontation du possible et de l'impossible que nous élargissons le champ de nos possibilités » écrit Bachmann dans le discours Die Wahrheit ist dem Menschen zumutbar ${ }^{42}$. Loin du mythe du génie qui « donne naissance à l'œuvre comme événement en coupant paradoxalement avec toute généalogie, toute genèse et tout genre ", tel que le réactualise Jacques Derrida ${ }^{43}$, Bachmann met en garde contre toute solution qui prétendrait inventer une nouvelle humanité ou communauté en dehors du Geschlecht, de cette part de nature qui relie les hommes entre eux ainsi qu'au monde autour d'eux.

\section{NOTES}

1. «Je me reproduis avec les mots et je reproduis aussi Ivan, j'engendre un genre nouveau, de mon union avec Ivan vient au monde ce que Dieu a voulu », Malina, in: Ingeborg Bachmann, Werke, hrg. von Christine Koschel, Clemens Münter, Inge von Weidenbaum, Piper Verlag München, tome III, p. 104. Traduction F. Rétif.

2. La touche d'humour dont elle est empreinte n'en disqualifie pas la valeur, au contraire.

3. La traduction révisée par Claire de Oliviera parue aux Éditions du Seuil n'a pas modifié dans ce cas l'originale de Philippe Jaccottet et en est resté au mot « race » particulièrement malvenu (voir p. 87).

4. Utilisé en outre pour traduire « Menschengeschlecht »/genre humain.

5. L'histoire du terme et du concept gender commence par la publication aux États-Unis de Sex and gender par Robert Stoller en 1968 et se poursuit par l'étude plus largement divulguée de la sociologue Ann Oakley, Sex, Gender and Society, en 1972.

6. Malina, op. cit., p. 104. Trad. F. Rétif.

7. Werke, op. cit., tome II, p. 212.

8. Nouvelle Alles, in : ibid., p. 143.

9. Nouvelle Das dreißigste Jahr, in : ibid., p. 132.

10. Dans le cadre de cet article, nous n'aborderons que marginalement la problématique des mythes, extrêmement vaste et à laquelle nous avons déjà consacré plusieurs contributions.

11. Voir interview du 23 mars 1971, in: Wir müssen wahre Sätze finden. Gespräche und Interviews, herausgegeben von Christine Kochel und Inge von Weidenbaum, Piper Verlag München, 1983, p. 92.

12. Voir Françoise Rétif, Simone de Beauvoir et Ingeborg Bachmann : Tristan ou l'Androgyne?, Bern, Francfort, New York, Peter Lang, 1989, première partie.

13. Id. ibid., deuxième partie.

14. Jean-Paul Sartre, L'Être et le Néant, Paris, Gallimard, 1943, collection Tel, p. 311. 
15. Cf. Nachlaß «Eine lange Nacht», Bibliothèque Nationale autrichienne, Nachlaß-Zahl 801. Trad. F. Rétif.

16. Cf. Simone de Beauvoir et Ingeborg Bachmann : Tristan ou l'Androgyne ?, op. cit., p. 69-75.

17. Cf. la nouvelle Undine geht, in : Werke, tome II, p. 253.

18. Fouqué s'inspire lui-même, entre autres, de Paracelse.

19. Geneviève Fraisse, "Le Chant des sirènes", in : La Controverse des sexes, Paris, PUF, coll. «Quadrige », 2001, p. 129.

20. Hélène Cixous, «Au temps d'Anna », Europe, n892-893, 2003, p. 81.

21. Werke, tome II, p. 254.

22. Cf. Gespräche und Interviews, op. cit., p. 46.

23. Les poèmes évoqués ici se trouvent dans le tome 1 de Werke. On peut trouver leur traduction française, faite par F. Rétif, dans le numéro de la revue Europe, voir note 20.

24. « Mein Teil, es soll verloren gehen », cf. Werke, tome I, p. 173.

25. Cf. Blanchot L'Entretien infini et Barthes le cours du Collège de France sur Le Neutre.

26. Werke, tome I, p. 168.

27. Idem.

28. Cf. Goethe, Trois contes et une nouvelle, Paris, Éditions Corti, coll. « Romantique », 1995.

29. Werke, I, p. 167.

30. Cf. Poème « Freies Geleit (Aria II) », in : Werke, I, p. 161.

31. Wittgenstein, Tractatus logico-philosophicus.

32. En particulier la phrase : «In allem Er-gründen und Be-gründen finden wir uns auf dem Weg zum Grund ».

33. Cf. Werke, op. cit., tome I, p. 167-168.

34. Souligné par nous.

35. Jean-Luc Nancy, Être singulier pluriel, Paris, Galilée, 1996, p. 30.

36. Jean-Luc Nancy, La Communauté désœuvrée, Paris, Christian Bourgois Éditeur, 1986 (rééd. 1990, 1999), p. 83.

37. In : Werke, op. cit., tome II. Voir pour d'autres exemples Françoise Rétif, Ingeborg Bachmann, Paris, Belin, 2008, chapitre 5.

38. Ibid., p. 153.

39. Ibid., p. 143 et 149.

40. Ibid., p. 138 et 158.

41. Ibid., p. 149.

42. Werke, op. cit., tome 4, p. 276. Traduction dans Europe, op. cit., p. 38.

43. Jaques Derrida, Genèses, généalogies, genres et le génie, Paris, Galilée, 2003, p. 55.

\section{RÉSUMÉS}

Comme l'atteste la phrase «Ich pflanze mich fort mit den Worten und ich pflanze auch Ivan fort, ich erzeuge ein neues Geschlecht, aus meiner und Ivans' Vereinigung kommt das Gottgewollte in die Welt» du roman Malina ou encore certaines nouvelles, Ingeborg Bachmann n'a jamais renoncé à la charge sexuelle, biblique et symbolique du mot « Geschlecht ». Son œuvre dans son ensemble oscille sans cesse entre Geschlecht et gender, entre construction et déconstruction de la différence des sexes. Ni construction ni déconstruction, sa déconstruction consiste peut-être en 
cette oscillation de la pensée qui ne se laisse pas fixer, sinon dans le refus de la violence et de l'inégalité entre les sexes. Loin du mythe du génie qui «donne naissance à l'œuvre comme événement en coupant paradoxalement avec toute généalogie, toute genèse et tout genre ", tel que le réactualise Jacques Derrida, Bachmann met en garde contre la chimère d'une humanité ou communauté qui prétendrait rompre avec le Geschlecht et la généalogie.

Wie sich in dem Satz « Ich pflanze mich fort mit den Worten und ich pflanze auch Ivan fort, ich erzeuge ein neues Geschlecht, aus meiner und Ivans' Vereinigung kommt das Gottgewollte in die Welt » aus dem Roman Malina, bzw sich auch in einigen Erzählungen bestätigt, hat Ingeborg Bachmann nie auf die sexuelle, biblische und symbolische Konnotation des Wortes « Geschlecht » verzichtet. Ihr Werk pendelt zwischen Geschlecht und Gender, zwischen Konstruktion und Dekonstruktion der Geschlechterdifferenzen ständig hin und her. Weder Konstruktion noch Dekonstruktion, ihre Dekonstruktion besteht in nichts anderem als in dem Oszillieren eines Denkens, das sich nicht festlegen lässt, es sei denn in der Ablehnung jeglicher Gewalt und Ungleichheit zwischen den Geschlechtern. Weit entfernt vom Mythos des Genies, das „das Werk als Ereignis entstehen lässt, indem paradoxerweise mit jeder Genealogie, jeder Genese oder jedem Geschlecht gebrochen wird“, so wie ihn Jacques Derrida reaktualisiert hat, warnt Bachmann vor dem Heraufbeschwören einer Menschheit oder Gemeinschaft, die mit der Genealogie brechen würde.

As confirmed in the novel Malina by the sentence "Ich pflanze mich fort mit den Worten und ich pflanze auch Ivan fort, ich erzeuge ein neues Geschlecht, aus meiner und Ivans' Vereinigung kommt das Gottgewollte in die Welt" and in some short stories, Ingeborg Bachmann never denied the sexual, biblical and symbolic connotation of the word Geschlecht. Her whole work fluctuates constantly between Geschlecht and Gender, between construction and deconstruction of sexual difference. Neither a construction nor a deconstruction, her deconstruction maybe consists in the oscillation of this thought, which holds out against any determination, excepting a refusal of violence and sexual discrimination. Far from Derrida's redefinition of the myth of the genius which generates the work as unpredictable experience of the event, and thus as the interruption of genealogical, genetic and generic ways of thinking literature and language (in Geneses, Genealogies, Genres and Genius), Bachmann warns against the pipe-dream of a humankind or a community intending to a break with Geschlecht and genealogy.

\section{AUTEUR}

\section{FRANÇOISE RÉTIF}

Université de Haute-Normandie - Rouen 